\title{
Noninvasive Respiratory Management of Patients With Neuromuscular Disease
}

\author{
John R. Bach, MD \\ Department of Physical Medicine and Rehabilitation for Rutgers New Jersey Medical School \& Center \\ for Ventilator Management Alternatives at University Hospital, Newark, NJ, USA
}

\begin{abstract}
This review article describes definitive noninvasive respiratory management of respiratory muscle dysfunction to eliminate need to resort to tracheotomy. In 2010 clinicians from 22 centers in 18 countries reported 1,623 spinal muscular atrophy type 1 (SMA1), Duchenne muscular dystrophy (DMD), and amyotrophic lateral sclerosis users of noninvasive ventilatory support (NVS) of whom 760 required it continuously (CNVS). The CNVS sustained their lives by over 3,000 patient-years without resort to indwelling tracheostomy tubes. These centers have now extubated at least 74 consecutive ventilator unweanable patients with DMD, over 95\% of CNVS-dependent patients with SMA1, and hundreds of others with advanced neuromuscular disorders (NMDs) without resort to tracheotomy. Two centers reported a $99 \%$ success rate at extubating 258 ventilator unweanable patients without resort to tracheotomy. Patients with myopathic or lower motor neuron disorders can be managed noninvasively by up to CNVS, indefinitely, despite having little or no measurable vital capacity, with the use of physical medicine respiratory muscle aids. Ventilator-dependent patients can be decannulated of their tracheostomy tubes.
\end{abstract}

Keywords Assisted cough, Glossopharyngeal breathing, Intermittent abdominal pressure ventilator, Mechanical insufflation-exsufflation, Neuromuscular diseases, Noninvasive ventilatory support

\section{INTRODUCTION}

Physical medicine respiratory muscle aids are methods and devices that apply pressures to the body and/or the airways to assist inspiratory and expiratory muscle function to noninvasively maintain normal alveolar ventilation and to optimize cough flows to preserve lung health. Body ventilators and manual thrusts apply positive and negative pressures to the body whereas manual resuscita- tors, ventilators, and mechanical insufflation-exsufflation (MIE) devices apply positive and negative pressures to the airways.

\section{HISTORICAL OBSERVATIONS AND "THE FIRST STEP TO WISDOM"}

In the winter of 1977-1978 as a first year resident physician in physical medicine and rehabilitation, I was

Received June 15, 2017; Accepted July 19, 2017

Corresponding author: John R. Bach

Department of Physical Medicine and Rehabilitation for Rutgers New Jersey Medical School \& Center for Ventilator Management Alternatives at University Hospital B-261, 150 Bergen street, Newark, NJ 07103, USA. Tel: +1-973-972-2850, Fax: +1-973-972-5725, E-mail: bachjr@njms.rutgers.edu ORCID: John R. Bach (http://orcid.org/0000-0002-4964-665X).

(c) This is an open-access article distributed under the terms of the Creative Commons Attribution Non-Commercial License (http://creativecommons.org/ licenses/by-nc/4.0) which permits unrestricted noncommercial use, distribution, and reproduction in any medium, provided the original work is properly cited. Copyright ( 2017 by Korean Academy of Rehabilitation Medicine 
assigned to the Goldwater Memorial Hospital (GMH) ventilator unit for patients with ventilatory pump failure due to neuromuscular disorders (NMDs). The GMH was a chronic rehabilitation hospital on Roosevelt Island in the East River in New York City. Driving to the hospital, on an almost daily basis, I passed GMH residents in motorized wheelchairs who were ventilating their lungs by receiving continuous noninvasive intermittent positive pressure ventilatory support (CNVS) via $15 \mathrm{~mm}$ angled mouthpieces (Fig. 1). They were traveling from GMH to the island's Main Street, often on their own, to see friends and go shopping.

It was my responsibility to write daily medical progress notes on each patient, many of whom had been medically stable there since 1954 . This took imagination. Remarkably, some of the GMH acute poliomyelitis survivors (PPSs) of the 1940s and 1950s are still alive today and using CNVS [1]. Many PPSs and high level spinal cord injured (SCI) patients quit iron lungs in 1954 in favor of mouthpiece CNVS and were never again hospitalized for respiratory difficulties. In 1977 about 40 of them left GMH for apartments in New York City and manage their own personal care attendants (PCAs) which many continue to do today. Most of these CNVS users have had little or no active muscle movement below their necks and no ability to breathe without CNVS from their portable mechanical ventilators except by glossopharyngeal breathing (GPB).

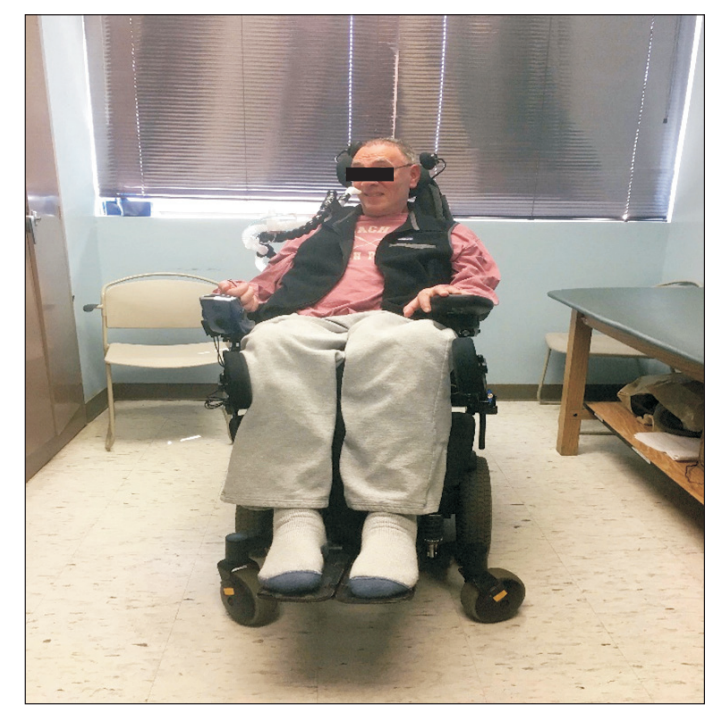

Fig. 1. A 61-year-old man with ALS dependent on sleep nasal noninvasive ventilatory support since 2014 and on daytime NVS via $15 \mathrm{~mm}$ angled mouthpiece as well, seen here, since February 2016.
They survived using daytime mouthpiece NVS (Fig. 1) and lipseal NVS for sleep (Fig. 2) although today most use nasal NVS for sleep.

The Thompson Bantam ventilator, on the market in the United States since 1957, could only deliver pressure preset control ventilation. Portable volume preset ventilators were not to be available until 1976. The over $250 \mathrm{GMH}$ NVS users with advanced NMDs were receiving air at 18 to $30 \mathrm{cmH}_{2} \mathrm{O}$ pressures irrespective of their vital capacities (VCs) or autonomous tidal volumes. This included patients with no VC. It was clear that lung insufflation pressures over $15 \mathrm{~cm} \mathrm{H}_{2} \mathrm{O}$ were needed to rest respiratory muscles and provide ventilatory support for patients with little or no VC. Without CNVS these patients would either still be in iron lungs around-the-clock or, more likely, would have undergone tracheotomies and by now be dead from complications related to the tube since four out of five patients with some NMDs using tracheostomy mechanical ventilation (TMV) die from tube related complications [2]. Besides prolonging survival, noninvasive management also preserves quality of life. Further, since all children under age 18 with tracheostomy tubes, even those having no physical disabilities or ventilator use, are 'entitled' to 16 hours per day of nursing care and TMV users are entitled to 16 to 24 hours per day of nursing care at $\$ 380,000$ to over $\$ 500,000$ per year, cost savings in the United States can exceed $\$ 400,000$ per year per individual CNVS user. Despite all the advantages of noninvasive



Fig. 2. A 68-year-old woman, spinal cord injured at birth, began sleep noninvasive ventilatory support via lipseal, seen here, in 1977 and began to require daytime mouthpiece NVS in 2004 and continuous NVS since 2010. 
management, fear of liability, 'paradigm paralysis' [1], and especially financial incentives to physicians, hospitals, long-term acute care facilities, nursing homes, home care nursing agencies, and even the US medical insurance industry prevent a paradigm shift away from unnecessary tracheotomies and invasive management.

From 1981 through 1989 I used the abbreviations 'MPPV' and 'MIPPV' for intermittent positive pressure ventilation delivered via simple mouthpieces during daytime hours or mouthpiece with lip cover phalange for sleep ventilatory support. Many of the hundreds of patients using MIPPV could not have survived more than minutes without using it. No randomized double blind placebo controlled studies demonstrating its effectiveness for prolonging survival were, therefore, possible to satisfy 'evidence-based criteria' for its efficacy [3]. Ironically, authors and article reviewers, many of whom who never consider that 'NIV' can provide 'ventilatory support,' do not understand that placebo controlled studies cannot be performed for interventions that replace vital bodily functions and actually question if the benefits of 'NIV' are overstated without controlled studies [4].

When I first described noninvasive ventilatory support via nasal access in 1987 [5], I used the term 'NIPPV' for this and continued using both 'NIPPV' and 'MIPPV' until 2002 when journal reviewers of my articles insisted that I use the common abbreviation 'NIV' for 'noninvasive ventilation. This 'NIV' term was being used by sleep physicians treating obstructive sleep apnea syndrome for patients with essentially normal respiratory muscle function. It was being used to refer to continuous positive airway pressure (CPAP) and low spans of bi-level PAP, both useless or inadequate for full respiratory muscle rest and ventilatory support. To distinguish my patients who were using noninvasive ventilatory support to survive, as opposed to NIV for obstructive apneas, in 2012 I qualified 'NIV' as 'continuous NIV for ventilatory support' [6] and 'full-setting, continuous ventilatory support by noninvasive intermittent positive pressure ventilation (NIV)' [7]. However, this confuses intermittent positive pressure ventilation, which is used for ventilatory support, with bi-level PAP which isn't intermittent but continuous air delivery and isn't conventionally used for ventilatory support. This brings to mind the ancient Chinese proverb that "the first step to wisdom is to call something by what it actually is." Therefore, also in 2012, because patients with NMDs are inappropriately evaluated and treated by NIV instead of by noninvasive ventilatory support or 'NVS' I insisted on publishing 'NVS' to distinguish it from the NIV used for sleep disordered breathing [8]. The necessity for this abbreviation is borne out by the fact that no publications other than my own cite 'ventilatory support' as a benefit of NIV for patients with NMDs.

\section{PATHOPHYSIOLOGY}

There are three respiratory muscle groups: the inspiratory muscles, the expiratory (for coughing, predominantly abdominal), and the bulbar-innervated muscles that protect the airways and permit glossopharyngeal breathing (GPB) and active lung volume recruitment (LVR). All three progressively weaken in NMDs. Inspiratory and expiratory muscle function, however, can be completely supported such that even patients with no measurable VC have now been using CNVS for up to 63 years [9], never to need tracheostomy tubes, but there are no noninvasive measures to substitute for bulbar-innervated muscles. However, even total paralysis of bulbar-innervated muscles does not indicate need for tracheotomy since patients can be positioned to drool rather than to aspirate (Fig. 3). The only indication for tracheotomy is the inability to expulse aspirated saliva or other airway debris to the extent that ambient air oxyhemoglobin saturation $\left(\mathrm{O}_{2}\right.$ sat) decreases below $95 \%$ because of central nervous system or upper motor neuron hypertonicity closing the upper airway too much for MIE to be effective [10,11]. The only NMD that this is seen in is ALS [12].

Physicians rarely distinguish ventilatory from respiratory failure. They evaluate them both by pulmonary function testing designed only for lung and airways diseases and treat them with CPAP or low span bi-level PAP. This results in needless morbidity and mortality, excessive expense, and decreased quality of life for patients with ventilatory pump failure. For pump failure patients, ventilatory insufficiency is initially nocturnal only. Normally VC does not decrease by more than $7.5 \%$ when going from sitting to supine [13]. Larger decreases that often result in orthopnea are caused by diaphragm dysfunction. Very often patients lose all ventilator free breathing ability when supine long before they require daytime ventilatory assistance (NVS). Many patients with ventilatory insufficiency survive despite orthopnea for years despite severe 


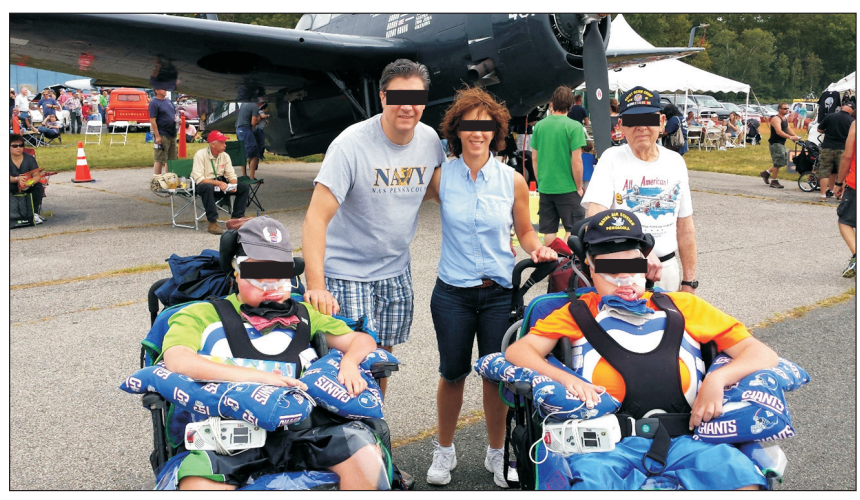

Fig. 3. Twenty- and 19-year-old brothers with severe spinal muscular atrophy type 1 , continuously dependent on noninvasive ventilatory support since 8 and 4 months of age, respectively, with no measurable vital capacities. The patients have received all nutrition via nasogastric tubes placed at 4 months of age. They drool but do not aspirate.

hypercapnia that can eventually result in cor pulmonale and symptoms of $\mathrm{CO}_{2}$ narcosis. Bicarbonate retention by the kidneys compensates for the effect of $\mathrm{CO}_{2}$ retention on blood $\mathrm{pH}$ but this also depresses central ventilatory drive and allows the brain to accommodate to hypercapnia without overt symptoms of acute ventilatory failure. Supplemental $\mathrm{O}_{2}$ administration further exacerbates hypercapnia by ventilatory depression and by the Haldane effect by which oxygenation of hemoglobin dissociates hydrogen ions from it, shifting the bicarbonate buffer equilibrium to $\mathrm{CO}_{2}$ formation. The $\mathrm{CO}_{2}$ released from red blood cells exacerbates hypercapnia. The hypercapnia associated with $\mathrm{O}_{2}$ therapy commonly results in ventilatory arrest. If, on the other hand, NVS is administered instead of $\mathrm{O}_{2}$, symptoms can dissipate, blood gases return to normal, excess bicarbonate ions are excreted, and ventilatory drive normalizes.

Another important reason that patients with NMD are inadequately treated is fear of the barotrauma that is commonly seen by using ventilatory assistance for patients with adult respiratory distress syndrome (ARDS). Intubated patients can generally require delivered volumes of $15 \mathrm{~mL} / \mathrm{kg}$ but this can result in high transpulmonary pressures and pneumothoraces in patients with intrinsic lung disease. Volumes of $7 \mathrm{~mL} / \mathrm{kg}$ result in less barotrauma in this population but at the cost of greater hypercapnia. Some now recommend $5 \mathrm{~mL} / \mathrm{kg}$ that permits even greater hypercapnia. While this approach can be beneficial for some patients with ARDS it is not for patients with NMD who have healthy lungs but impaired respiratory muscle function. The under ventilated lungs and chest walls of people with NMD can stiffen and shrink; symptomatic hypercapnia develop; and the decreased pulmonary compliance can make it impossible to normalize alveolar ventilation and alleviate symptoms until an aggressive program of LVR (read below) at high lung insufflation pressures (typically 40 to $60 \mathrm{cmH}_{2} \mathrm{O}$ ) is instituted [14]. Also, interestingly, pulmonary compliance equations suggest that every $1 \mathrm{cmH}_{2} \mathrm{O}$ of insufflation pressure can provide $100 \mathrm{~mL}$ of air. This suggests that 6 $\mathrm{cmH}_{2} \mathrm{O}$ might be enough for a full tidal volume. However, during general anesthesia that includes neural paralyzing agents that reduce inspiratory capacity to $0 \mathrm{~mL}$, pressures of 15 to $25 \mathrm{cmH}_{2} \mathrm{O}$ are required for lung ventilation. Likewise, the hundreds of GMH patients with little to no $\mathrm{VC}$ also required, and many continue to require, 18 to $35 \mathrm{cmH}_{2} \mathrm{O}$ pressure preset ventilation. As lung volumes increase the intrapulmonary pressures generated by insufflated volumes increase exponentially rather than linearly. Thus, the conventional approach of extubating very weak NMD patients to bi-level PAP limited to spans of 4 to $8 \mathrm{cmH}_{2} \mathrm{O}$ is grossly inadequate.

\section{CONVENTIONAL LONG-TERM EVALUATION AND MANAGEMENT}

Patients are usually monitored by pulmonary function testing (PFTs), all of which designed to evaluate lung and airways rather than muscle function. The PFTs measure lung volumes, maximum expiratory flow rates, bronchodilator responses for bronchospasm, lung diffusion, plethysmography (chest and abdominal movements that can falsely denote obstructive apneas and hypopneas rather than chest muscle weakness), maximum expiratory pressures, and gas exchange such as by arterial blood gas analyses. None of these are very relevant for the patient with pure ventilatory pump failure who requires capnography, spirometry in various positions, cough peak flows, and oximetry.

Also typically in the United States, patients with progressive NMDs, even when asymptomatic, are sent for polysomnograms as their VCs decrease to $50 \%$ of predicted normal values. Polysomnograms, however, are programmed to interpret all apneas and hypopneas as 
central or obstructive in nature even when the problem is entirely respiratory muscle weakness. Mistakenly thinking that the problem is central and obstructive apneas and hypopneas or 'sleep disordered breathing (SDB),' sleep specialists attempt to titrate them away by administering CPAP. This must quickly fail, however, since CPAP does not assist inspiratory muscle function. Many patients are now placed on bi-level PAP but at only low spans (drive pressures), that is, inspiratory PAP (IPAP) minus expiratory PAP (EPAP) less than $10 \mathrm{cmH}_{2} \mathrm{O}$. While beneficial, this is suboptimal because low spans are neither adequate for optimal respiratory muscle rest nor for the full ventilatory support that often eventually becomes necessary. As a result of inappropriate titration for SDB patients with NMD have presented to us attempting to use bi-level PAP with IPAP over $20 \mathrm{cmH}_{2} \mathrm{O}$ and EPAP over $15 \mathrm{cmH}_{2} \mathrm{O}$. Besides the ineffective spans, this is tantamount to breathing in a tornado. Our center has now managed over 2,000 NVS users without polysomnography. Many hundreds have become CNVS dependent. Obviously, therefore, polysomnography, especially when $\mathrm{CO}_{2}$ monitoring is not included, is not needed to manage NMD.

Patients not referred to centers that specialize in definitive noninvasive management as listed in http://www. breatheNVS.com must eventually develop respiratory failure which will either be caused by airway mucus plugging due to ineffective cough flows or by hypercapnic coma due to oxygen $\left(\mathrm{O}_{2}\right)$ therapy. Conventionally managed patients are typically prescribed supplemental $\mathrm{O}_{2}$ and bronchodilators even though there is no evidence that either is beneficial. Bronchodilators, while usually not harmful, can cause anxiety, tachycardia, gastroenterological and other symptoms. Tachycardia can be problematic for patients with cardiomyopathies and cardiac dysrhythmias.

The supplemental $\mathrm{O}_{2}$ is a bigger problem. It depresses ventilatory drive and can both acutely and chronically exacerbate hypercapnia and increase $\mathrm{CO}_{2}$ levels by 50 to $100 \mathrm{mmHg}$ or more $[15,16]$. It also renders oximetry useless as a gauge of alveolar ventilation and airway secretion encombrement $[16,17]$. In the United States it is rare for a patient to present with $\mathrm{CO}_{2}$ narcosis and coma without receiving supplemental $\mathrm{O}_{2}$. In a study of 316 patients with NMD who required intubation for acute respiratory failure (ARF), all were intubated for acute deterioration that was preceded by $\mathrm{O}_{2}$ therapy prescribed by clinicians with no knowledge of the patient's $\mathrm{CO}_{2}$ levels [16]. In retrospect it was found that $\mathrm{CO}_{2}$ levels increased by a mean $52.1 \mathrm{mmHg}$, and at times by over $100 \mathrm{mmHg}$, in as little as 20 minutes with $\mathrm{O}_{2}$ administration [16]. Patients with NMD most commonly develop ARF and are intubated as a result of otherwise benign upper respiratory infections (URIs) developing into pneumonias because their cough flows are inadequate to clear airway mucus and emergency medical services administer $\mathrm{O}_{2}$ and/or low span bi-level PAP instead of NVS and MIE at optimal settings.

\section{PATIENT EVALUATION SPECIFIC FOR VENTILATORY PUMP FAILURE}

While patients with diminished ventilatory reserve who can walk complain of exertional dyspnea, wheelchair patients more likely complain of anxiety and inability to sleep. Eventually symptomatic hypercapnic patients present with morning headaches, fatigue, sleep disturbances, and hypersomnolence [18]. This often occurs when hypoventilation results in hypercapnia and hypoventilation sufficient for daytime $\mathrm{O}_{2}$ sat to decrease below $95 \%$. Symptoms may be minimal except during intercurrent URIs. Signs to observe for ventilatory insufficiency include orthopnea, tachypnea, paradoxical breathing, hypophonia, nasal flaring, use of accessory respiratory muscles, cyanosis, flushing or pallor, and airway congestion. Lethargy and confusion signal $\mathrm{CO}_{2}$ narcosis.

Spirometry, a peak flow meter, a capnograph, and an oximeter should be available for every outpatient visit. The VC is measured in sitting and supine positions. Orthopnea is often accompanied by a sitting/supine VC difference greater than $30 \%$, irrespective of absolute values. Because hypoventilation is worst during sleep, the supine VC is perhaps the most important indicator of ventilatory dysfunction and need to introduce nocturnal NVS. Spirometry is also useful for monitoring progress with GPB and active lung volume recruitment (air stacking). Air stacking is performed by receiving consecutively delivered volumes of air via a manual resuscitator. The glottis holds the delivered volumes without exhaling until the maximum volume is held. This is exhaled into a spirometer and is termed the maximum insufflation capacity (MIC). The extent to which the MIC exceeds VC (MIC-VC) objectively and reproducibly quantitates glot- 
tis, and therefore, bulbar innervated muscle function. Patients who learn GPB can often air stack consecutive GPB boluses to or beyond the MIC to a glossopharyngeal maximum single breath capacity (GPmaxSBC) [19]. A nasal or lip cover interface is used for air stacking when the lips are too weak for maximal air stacking via a simple mouthpiece. For patients wearing thoracolumbar braces the VC is measured both with the brace on and off. A well-fitting brace can increase $\mathrm{VC}$ and a poorly fitting one decrease it.

Cough peak flows (CPF) are measured via peak flow meter (Access Peak Flow Meter; Healthscan Products Inc., Cedar Grove, NJ, USA). CPF of $160 \mathrm{~L} / \mathrm{min}$ are approximately the minimum needed to cough effectively [17], but risk of URI triggered pneumonia increases as CPF decrease below $300 \mathrm{~L} / \mathrm{min}(5 \mathrm{~L} / \mathrm{s})$. Both unassisted CPF and manually assisted CPF are determined. The latter result from a deeply air stacked volume followed by an abdominal thrust concomitant with the air coughed into a peak flow meter. The manually assisted CPFs of patients with VCs greater than 1,500 mL may not be significantly increased by air stacking before the abdominal thrust.

With the availability of oximetry, and capnography to measure end-tidal $\mathrm{CO}_{2}$, arterial blood gas sampling is rarely if ever needed for people with NMD [20]. In addition to the discomfort, $25 \%$ of patients hyperventilate as a result of the anxiety and pain caused by the arterial puncture [21]. Noninvasive continuous blood gas monitoring; that is, by end-tidal $\mathrm{CO}_{2}$ (capnography) or transcutaneous $\mathrm{CO}_{2}$ and oximetry, usually yield more useful information.

Irrespective of laboratory values, all clearly symptomatic patients with decreased supine VC require a trial of NVS to ease symptoms. If symptoms are questionable, however, nocturnal capnography and oximetry monitoring can be helpful to convince the patient to try NVS and the third party payer to pay for it. $\mathrm{CO}_{2} \mathrm{~s}$ approaching $50 \mathrm{mmHg}$ and $\mathrm{O}_{2}$ sats dipping frequently below $95 \%$ can signal hypoventilation and potential symptomatic benefit from sleep NVS. The oximeter and the capnography should be capable of overnight data collation. The studies are performed in the patient's home. Any questionably symptomatic patient with decreased VC, multiple nocturnal $\mathrm{O}_{2}$ desaturations (desats) below 95\%, and elevated nocturnal $\mathrm{CO}_{2}$ should try nocturnal NVS. Because only patients improperly treated with supplemental $\mathrm{O}_{2}$ tend to develop $\mathrm{CO}_{2}$ narcosis, and ARF is usually caused by ineffective coughing during URIs, patients who find that NVS is more burdensome than beneficial can safely discontinue it until next reevaluation.

Symptomatic patients with good muscle strength, normal supine VC, and no significant sleep $\mathrm{O}_{2}$ desat or $\mathrm{CO}_{2}$ retention are evaluated for SDB by polysomnography. Polysomnography is not indicated for patients with decreased VC as it invariably results in inappropriate treatment with $\mathrm{O}_{2}$, CPAP, and low-span bi-level PAP instead of NVS. Further, treatment of asymptomatic NMD patients on the basis of polysomnographic abnormalities neither significantly prolongs life nor improves its quality.

\section{GLOSSOPHARYNGEAL BREATHING}

Both inspiratory and expiratory muscle function can be assisted by GPB $[19,22]$. GPB can provide patients with no VC or breathing tolerance with normal alveolar ventilation without using a ventilator. It provides security in the event of sudden ventilator failure, day or night [23-25]. Using GPB the glottis pistons boluses of air into the lungs to supplement an autonomous breath. The glottis closes to hold each bolus. One GPB typically consists of six to nine boluses of 40 to $200 \mathrm{~mL}$ each [26,27] (Fig. 4). During the training period, GPB efficiency is monitored spirometrically as the milliliters of air per bolus, GPB boluses per breath, and breaths per minute. A training manual [23] and videos are available [24,25].

Seventy per cent of high level SCI patients, PPSs, and others with good bulbar innervated muscle function can add to maximum inspiratory efforts by GPB. Approximately $60 \%$ of CNVS users with no autonomous ability to breathe but good bulbar muscle function can use GPB instead of CNVS up to all day long [14,28]. On the other hand, GPB is rarely useful in the presence of an indwelling tracheostomy tube so TMV users are always afraid of accidental ventilator disconnections or sudden ventilator failure. Although severe oropharyngeal muscle weakness limits GPB effectiveness we have managed 13 DMD CNVS users who had no breathing tolerance other than by GPB [22]

The safety and versatility provided by GPB are principal reasons to decannulate patients of their tracheostomy tubes in favor of noninvasive management. With usually intact bulbar musculature high-level SCI patients are among the most ideal candidates to be decannulated to 


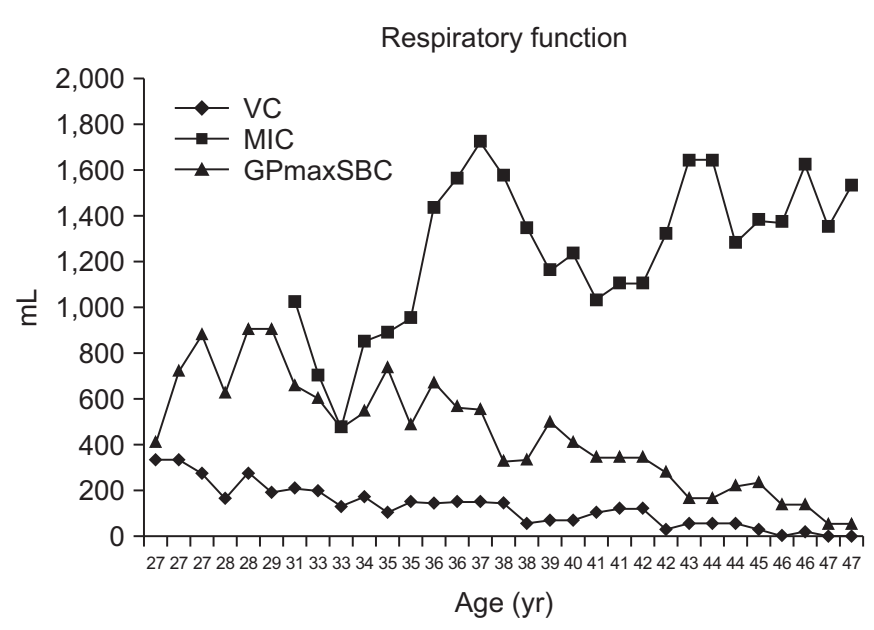

Fig. 4. Changes in vital capacity (VC), maximum insufflation capacity, and glossopharyngeal maximum single breath capacity over a 20 year period for a patient with Duchenne muscular dystrophy. The GPB, added to his autonomous tidal volumes, permitted the patient to breathe without requiring full ventilatory support until 43 years of age despite having a VC of $20 \mathrm{~mL}$ from age 42 . VC, vital capacity; MIC, maximum insufflation capacity; GPmaxSBC, glossopharyngeal maximum single breath capacity.

CNVS and to master GPB for ventilator free breathing but because of strong financial incentives for conventional invasive management they are perhaps the least likely to benefit from this. No SCI patients with intact bulbar innervated musculature are safer with tracheostomy tubes than without them. Mouthpiece NVS, intermittent abdominal pressure ventilation, phrenic or diaphragm pacing can be used for daytime support and nasal or oronasal NVS for sleep. With the tracheostomy tube out there will be no daily airway secretions to clear and manually assisted coughing can be effective [14,28].

\section{LUNG VOLUME RECRUITMENT}

The goal of LVR is to maintain lung and chest wall compliance, to promote normal lung and chest wall growth for children, and to maximize lung inflation to approach predicted inspiratory capacities. Pulmonary compliance diminishes when a patient cannot expand the lungs to normal values. When the largest breath one can take is only a fraction of predicted inspiratory capacity the lungs and chest wall stiffen and shrink. Regular mobilization by expanding the lungs to approach predicted inspira- tory capacity is required to prevent chest wall contractures and lung and chest wall restriction. This can only be achieved by LVR and to a degree by nocturnal NVS for infants who cannot perform active LVR [29].

Air stacking, which is an active LVR, can increase VC and CPF and diminish atelectasis [26,27]. The deep lung volumes by air stacking also permit patients to raise voice volume and speak longer phrases. Because any patient who can air stack is also able to use NVS, if intubated for ARF he or she can more easily be extubated directly to CNVS regardless of absence of any ventilator free breathing ability.

Some recommend instituting daily air stacking once the VC is less than $\mathbf{8 0 \%}$ of normal [30]. Active LVR is air stacking via a mouthpiece, lip cover, nasal, or even an oronasal interface. What is important is that the glottis hold consecutively delivered air volumes to the MIC. Although the VC may decrease with advancing disease, the MIC tends to increase by maximal air stacking 10 to 15 times at least two or three times daily [27]. The air is usually delivered by manual resuscitator or volume preset ventilator. Because of the importance of air stacking we preferentially prescribe volume rather than pressure preset ventilation via active ventilator circuits, that is, circuits with exhalation valves.

Passive LVR is provided by consecutively delivering volumes of air via a manual resuscitator with the exhalation valve blocked so that the patient cannot exhale until a maximum volume is comfortably attained. This is appreciated by feeling the increasing resistance of lung recoil while squeezing the manual resuscitator to inflate the lungs. The air can also be delivered using the insufflation mode of MIE. For this, the pressure must be set to at least 55 to $60 \mathrm{cmH}_{2} \mathrm{O}$. These pressures are low by comparison with the pressures attained by many patients who have been performing air stacking three times daily for over 50 years without clinically apparent barotrauma. We have had only one case of chronic pneumothorax in more than 2,000 air stacking plus NVS users [31].

Since infants cannot air stack or cooperate with active LVR, all small children with paradoxical chest wall movement are placed on nocturnal NVS to reverse the paradoxing and to prevent or reverse pectus excavatum as well as to promote lung growth. The ventilatory assistance provided by sleep nasal NVS also relieves the typically observed flushing, perspiration, and deranged 
sleep [29]. Low span bi-level PAP does not reverse paradoxical breathing, only NVS does. In addition to nocturnal NVS, passive LVR can be provided from a manual resuscitator via an oral-nasal interface by timing the air delivery to the child's inhalations. The children can also be placed on the Cough-Trak or auto-triggering mode of the CoughAssist (Philips-Respironics Inc., Murrysville, PA, USA). Children can become cooperative with deep insufflation therapy by 14 to 30 months of age. The maximum passive insufflation volume can be measured and is termed the 'lung insufflation capacity' [32].

\section{NONINVASIVE POSITIVE PRESSURE VENTILATORY SUPPORT}

Normal alveolar ventilation should and can be maintained around-the-clock irrespective of extent of respiratory muscle dysfunction. This used to be done by using negative pressure body ventilators (NPBVs) but these cause obstructive sleep apneas that can necessitate CPAP. The use of NVS obviates the need for both the body ventilators and the CPAP and is far more comfortable and effective [33]. Blood gases improve dramatically when patients are switched from body ventilators to NVS [34].

\section{DAYTIME SUPPORT}

The intermittent abdominal pressure ventilator, or BachBelt (Dima Italia Inc., Bologna, Italy), operates by about $2.5 \mathrm{~L}$ of air being delivered from a powerful portable ventilator into an elastic air sac that is incorporated into a girdle or corset worn under the clothing (Fig. 5). The sac is cyclically inflated in this manner, moving the diaphragm upwards, and with cessation of airflow and emptying of the sac the diaphragm descends due to gravity, causing air to enter the upper airways and lungs. A trunk angle of $30^{\circ}$ or more from the horizontal is necessary for it to be effective. If the patient has any inspiratory capacity or is capable of GPB, GPB adds volumes of air to those taken in mechanically. The intermittent abdominal pressure ventilator can augment tidal volumes by $300 \mathrm{~mL}$ to $1,200 \mathrm{~mL}$ and patients with less than 1 hour of ventilator free breathing tolerance often prefer it to using NVS during daytime hours [35]. A new non-pneumatic system using a small motor to move a baffle against the abdomen is also in production [36].

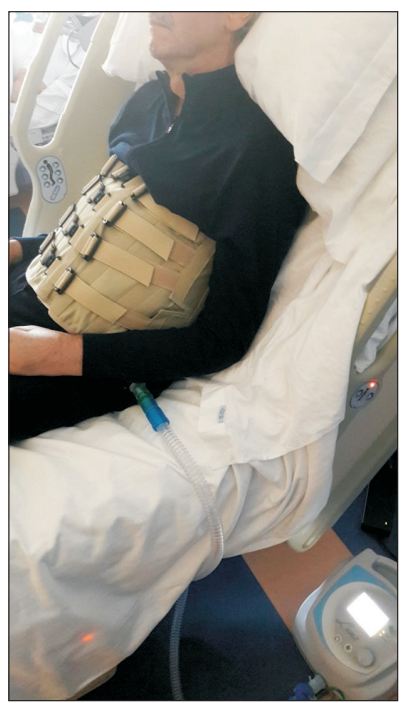

Fig. 5. The intermittent abdominal pressure ventilator (BachBelt, Dima Italia Inc., Bologna, Italy), seen here, is normally worn under clothing and, therefore, optimizes appearance.

Although patients with NMD usually begin NVS for sleep to treat symptoms of sleep hypoventilation, many first use it during daytime hours to facilitate eating, speaking louder, and coughing. As inspiratory muscles weaken respiratory rate increases and often exceeds 40 per minute. This leaves only about 1 second for swallowing food. Although foods can only be safely swallowed during an expiratory pause, with tachypnea and dysphagia, unsafe inspiratory pause swallowing occurs and many patients avoid eating and lose weight as a result. We generally introduce NVS in the clinic by setting up a portable ventilator to deliver volumes of $800 \mathrm{~mL}$ to more typically 1,200 to $1,500 \mathrm{~mL}$ with a high back-up rate. Since normal minute ventilation is about $6,000 \mathrm{~mL}$, even patients with $0 \mathrm{~mL}$ of $\mathrm{VC}$ only need to grab the mouthpiece four times a minute for normal minute ventilation and this allows about 15 seconds for swallowing. Many patients gain weight as a result.

The optimally managed patient with advancing NMD becomes CNVS dependent without ever being hospitalized for invasive airway tubes. When progressively weakening patients become dyspneic when discontinuing NVS in the morning they are switched from nasal or oronasal interfaces to NVS via a mouthpiece. The NVS via 15 $\mathrm{mm}$ angled mouthpieces is the most important method of daytime NVS. Some patients keep the mouthpiece 
between their teeth all day [33]. Most, however, have the mouthpiece fixed adjacent to the mouth as when eating. A metal clamp or microphone holder attached to a wheelchair achieves this. The mouthpiece can also be fixed onto sip and puff, chin, or tongue controls of motorized wheelchairs. The Trilogy ventilator (Philips Respironics Inc.) is now available with an attached flexible support arm that holds the mouthpiece next to the mouth but this is more expensive than simple flexible metal support arms that are readily available to hold microphones.

Setting the ventilator for the relatively large delivered volumes of 800 to $1,500 \mathrm{~mL}$ for daytime support permits the user to take as much of the air as desired, and, thereby, physiologically vary tidal volumes and air stack ad lib. The Trilogy also has a Kiss Trigger that permits air delivery by simply touching (grabbing) the mouthpiece with the lips so that back-up rates can be conveniently set to zero. The variable tidal volumes facilitate changing speech volumes and length, cough flows, and air stacking (active LVR) to promote lung health.

Some neck movement and lip function are needed for effective mouthpiece NVS. The soft palate also needs to move posteriocranially to seal off the nasopharynx from leak out of the nose. The glottis must also open reflexively to breathe in the air. These normally reflex movements can take time to relearn for CNVS users after decannulation from CTMV since when using TMV with an inflated cuff the glottis remains closed [28].

While nasal NVS is most practical for use during sleep, it is also indicated for infants and for those who cannot grab or retain a mouthpiece because of oral and neck muscle weakness and inadequate jaw opening. As such, daytime nasal CNVS is a viable and desirable alternative to tracheostomy and always preferred by patients over resort to tracheotomy. When using nasal NVS around-theclock, however, the nasal interfaces must be alternated to avoid excessive skin pressures and decubiti. We typically use nasal prongs and nostril covering systems during daytime hours and triangular 'mask' designs for sleep.

\section{NOCTURNAL SUPPORT}

Typical symptoms of fatigue, hypersomnolence, morning headaches, and depression as well as daytime hypercapnia and $\mathrm{O}_{2}$ desat can be completely reversed by nocturnal NVS, at least temporarily, until further muscle weakening causes patients to become dyspneic when discontinuing it in the morning.

It is usually for sleep that symptomatic patients begin NVS. The NVS is delivered via lip cover, nasal, or oronasal interfaces at delivered volumes of 800 to $1,500 \mathrm{~mL}$ or pressures typically of 18 to $25 \mathrm{cmH}_{2} \mathrm{O}$ with physiologic back-up rate usually of 12 to $14 / \mathrm{min}$. Active ventilator circuits are used to permit volume preset ventilation for active LVR. Since patients take any portion of the set volumes overnight as well as during the day, the variable tidal volumes also facilitate communication by varying voice volumes as well as facilitating unassisted coughing overnight, and optimally resting inspiratory muscles [37].

The patient must open the glottis and vocal cords, dilate the hypopharynx, and maintain airway patency to receive NVS during sleep as well as when awake. Some nasal NVS users actually keep their mouths closed throughout sleep but this is not at all necessary. The NVS propels the soft palate forward against the posterior surface of the tongue to diminish air leakage out of the mouth [5]. However, even without this passive mechanism, excessive leakage is prevented by the alert though sleeping brain's ventilatory drive being undiminished by sedatives or $\mathrm{O}_{2}$ administration. Repeated brief arousals, of which the patient is unaware, cut off the leak and reverse $\mathrm{O}_{2}$ desats. Mouthpiece/lip cover and nasal NVS are open systems that necessitate such central nervous system reflexes to prevent excessive leakage during sleep [38]. All patients leak, however excessive leakage is only that which results in $\mathrm{O}_{2}$ desats severe enough to cause arousals of which the patient becomes aware.

There are numerous commercially available nasal interfaces (e.g., CPAP 'masks'), both vented, that is, with portals or open areas that permit leak when used with passive ventilator circuits for CPAP and bi-level PAP, and non-vented, that is, without holes or leaky areas when used with active circuits (circuits with exhalation valves). When used with an active circuit for volume or pressure preset ventilation, the portals of the vented interfaces must be covered otherwise non-vented interfaces should be used. Several interfaces should always be tried and the patient encouraged to alternate their use, especially when CNVS dependent. Excessive insufflation leakage causes dyspneic arousals for about $3 \%$ of patients [39]. These are avoided by switching from open to closed systems such as lip cover-nasal prong oronasal interfaces. 
Such interfaces deliver air via mouth and nose and require relatively low strap pressures, thereby optimizing skin comfort while minimizing air leakage. Excessive leakage is also prevented by sustaining ventilatory drive by maintaining normal daytime $\mathrm{CO}_{2}$ as well as by avoiding supplemental $\mathrm{O}_{2}$ and sedatives.

Suboptimal humidification dries out and irritates nasal mucous membranes, causes sore throat, and results in vasodilatation and nasal congestion. Increased airflow resistance to $8 \mathrm{cmH}_{2} \mathrm{O} / \mathrm{L}$ per second can be caused by the loss of humidity that is caused by unidirectional airflow with expiration via the mouth during nasal NVS [40]. This can be reduced by heated humidification using a hot water bath humidifier [40]. Decongestants can help relieve sinus irritation and nasal congestion. Switching to a lip cover-only interface can relieve sinus pressure, nasal congestion, and the nasal bridge pressure discomfort associated with nasal NVS.

Abdominal distention tends to occur sporadically in sleep NVS users. The air usually passes as flatus when the patient is mobilized in the morning. When severe, however, it can decrease VC and increase ventilator dependence. Patients using volume preset ventilation during sleep are more susceptible since transient glottis closure forces air under high pressure into the esophagus, stomach, and intestines. Patients for whom distention is a severe problem are switched to pressure preset ventilation. Normal gastroesophageal sphincter functional integrity is to a pressure of $25 \mathrm{cmH}_{2} \mathrm{O}$ but when lower, as is often the case in NMD, aerophagia and abdominal distension can occur even without ventilator use. When NVS is delivered to patients who have this problem, it usually exacerbates the distension and can mandate placement of an indwelling gastrostomy tube to burp out the air to permit use of NVS at all. Sometimes a rectal tube can be helpful to decompress the colon as well.

Although often described as a complication or limiting factor for 'noninvasive ventilation,' secretion encumbrance results from failure to use MIE effectively via both invasive and noninvasive interfaces. The use of NVS is relatively contraindicated by the presence of depressed cognitive function, orthopedic conditions interfering with noninvasive interfaces, pulmonary diseases necessitating a high fraction of inspired oxygen, and with uncontrolled seizures or substance abuse which would prevent reliable access to noninvasive interfaces [7]. Likewise, supplemental oxygen and sedatives can render sleep NVS ineffective.

Although in rare cases diaphragm and phrenic nerve pacing can provide adequate tidal volumes to ventilate the lungs of patients with high level SCI, these are never indicated for patients with NMDs. Lately diaphragm pacing has been promoted for patients with ALS but two international studies have demonstrated that it hastens death for these patients $[41,42]$. Likewise, we reported that rates of loss of VC are greatly accelerated by diaphragm pacing [6]. Even for high level SCI patients, CNVS following tube decannulation is a desirable alternative to electrophrenic and diaphragm pacing since unlike for pacing for which tracheostomy tubes are kept to allay pacer-caused obstructive apneas, decannulation and NVS permits patients to master GPB for ventilator free breathing ability.

\section{ASSISTED COUGHING}

\section{Manually assisted coughing}

Manually assisted coughing is done by applying an abdominal thrust timed to glottis opening once the lungs have a deep volume of air provided by air stacking. In one study, manually assisted CPF were $255 \pm 100 \mathrm{~L} / \mathrm{min}$ for subjects whose unassisted cough flows were $150 \pm 120 \mathrm{~L} /$ min [27]. The more effective flows can prevent intercurrent URIs from developing into pneumonias and ARF [17]. Patients with sufficiently functional bulbar-innervated musculature to air stack but who are unable to generate over $160 \mathrm{~L} / \mathrm{min}$ of assisted CPF usually have upper airway obstruction which needs to be evaluated by laryngoscopy for reversibility. Patients unable to air stack due to inability to close the glottis can still have cough flows assisted by abdominal thrust applied to a deep breath. Such flows are often less than $160 \mathrm{~L} / \mathrm{min}$.

\section{Mechanical insufflation-exsufflation}

Mechanically assisted coughing is simply the use of MIE. Although MIE was provided by the Cof-flator (OEM Company, Hartford, CT, USA) from 1953 through 1967, it was unavailable until I persuaded Mr. John H. (Jack) Emerson to remanufacture his In-exsufflator in 1988. It was for this reason that I dedicated my first book to him [43]. The Emerson In-exsufflator was approved by the US Food and Drug Administration in November 1992 and released 
onto the American market in February 1993. Prior to that our center's patients shared five Cof-flators that were obtained for us from a respiratory products museum in Seattle, Washington. Respironics Inc. bought the John H. Emerson Company, essentially their In-exsufflator, in 2007.

For about $20 \%$ of patients, and especially for patients who use MIE at less than optimal settings, the application of an abdominal thrust and sometimes a chest compression concomitant with the exsufflation phase of MIE significantly increases the MIE-exsufflation flows (MIE-EF) to increase its efficacy in expulsing airway debris.

Deep insufflations followed immediately by deep exsufflations at pressures of 40 to $-40 \mathrm{mmHg}\left(54.1 \mathrm{cmH}_{2} \mathrm{O}\right)$ are usually most effective and preferred. These pressures are applied via oronasal interfaces or simple mouthpieces. When used via a translaryngeal or tracheotomy tube, pressures of 60 to $70 \mathrm{cmH}_{2} \mathrm{O}$ are needed because of the pressure drop-off and diminution of air flows across the tubes [44]. Using MIE via invasive tubes is crucial for preparing patients for extubation or decannulation $[45,46]$. The tube cuffs, when present, should be inflated when using MIE.

Respironics Inc. evolved the In-exsufflator into the CoughAssist which can be manually or automatically cycled. Manual cycling facilitates caregiver-patient coordination of inspiration and expiration with insufflation and exsufflation. One treatment consists of about five cycles of MIE followed by a short period of unassisted breathing or ventilator use to avoid hyperventilation. Insufflation and exsufflation times at the 50 to $70 \mathrm{cmH}_{2} \mathrm{O}$ pressures are those necessary for maximum clinically observed chest expansion followed without pause to full clinical lung emptying without causing shortness of breath. In general, 2 to 3 seconds are needed for each. Longer times and pauses can cause shortness of breath. Treatment continues until no further secretions are expulsed and secretion-related $\mathrm{O}_{2}$ desats are reversed. Use can be required as often as every 20 to 30 minutes around the clock during chest infections or for 36 hours following extubation or decannulation.

The use of MIE via the upper airway can be effective for children as young as 11 months of age who on occasion become accustomed to it without crying or closing their glottises. At 2.5 to 5 years of age most children cooperate fully with it and may never again develop a URI- pneumonia. Small children and infants who do not cooperate with it can trigger it with each breath by using the Cough-Trak mode of the CoughAssist. Exsufflation-timed abdominal thrusts can often increase MIE-EF for infants as can be observed by using the E-70 and T-70 model CoughAssists that display these MIE-EF.

Whether via the upper airway or via invasive airway tubes, routine airway suctioning misses the left main stem bronchus about $90 \%$ of the time [47]. MIE, on the other hand, provides effective exsufflation flows in both left and right airways without the discomfort or airway trauma of tracheal suctioning. Patients prefer MIE to suctioning for comfort and effectiveness and find it less tiring [48]. Deep airway suctioning, whether via airway tube or the upper airway, can be discontinued for most patients. Also, the VC, pulmonary flow rates, and $\mathrm{O}_{2}$ sat, when abnormal, improve with clearing of airway secretions and mucus by MIE [49]. Increases in VC of $15 \%$ to $40 \%$ were reported for 67 patients with 'obstructive dyspnea,' and $55 \%$ increases in VC for patients with NMDs using MIE with no untoward effects [50]. We reported $15 \%$ to $400 \%$ (200-800 mL) improvements in VC and normalization of $\mathrm{O}_{2}$ sat using MIE for patients with NMD during chest infections [51]. The effective use of MIE is the major reason that we routinely extubate and decannulate ventilator unweanable patients. The only exceptions are patients with upper airway hypertonicity and collapse [11] due to central nervous system or upper motor neuron disease including about half of all patients with ALS. For the latter, MIE-EF deteriorate over time as upper airway patency diminishes. Once the flows decrease to about $100 \mathrm{~L} / \mathrm{m}$ or lower for them tracheotomy usually becomes necessary [10]. In general, patients who benefit most from MIE attain MIE-EF over $200 \mathrm{~L} / \mathrm{m}$. Such flows are typically attained for all patients with NMD except advanced ALS.

\section{OXIMETRY FEEDBACK PROTOCOL}

Although CTMV can extend survival for patients with NMD [52], morbidity and mortality outcomes are more favorable using CNVS so tracheotomies should be avoided or reversed [53,54]. Reversing the hypercapnia and $\mathrm{O}_{2}$ desat of alveolar hypoventilation, preventing pneumonia during chest URIs, or switching a patient from CTMV to CNVS is facilitated by oximetry feedback. An $\mathrm{O}_{2}$ sat alarm can be set at about $5 \%$ over the patient's baseline 
and with improvement, increased eventually to $94 \%$. The alarm signals the patient to increase and eventually normalize alveolar ventilation and $\mathrm{O}_{2}$ sat by taking deeper breaths or by using mouthpiece or nasal NVS for the deeper air volumes to increase or normalize $\mathrm{O}_{2}$ sat and $\mathrm{CO}_{2}$. With advancing NMD the patient eventually requires increasing periods of daytime NVS to maintain alveolar ventilation and can become CNVS dependent.

Continuous $\mathrm{O}_{2}$ sat feedback is especially important during URIs. The cough flows of infants and small children who never have the strength to sit are inadequate to prevent URI-triggered pneumonias and ARF. Patients use MIE for any dip in $\mathrm{O}_{2}$ sat below $95 \%$. When using CNVS, dips are usually caused by bronchial mucous plugging that, if not quickly cleared, result in atelectasis, pneumonia, and collapsed lungs. Thus, patients use NVS and MIE to avoid pneumonia and ARF by maintaining normal $\mathrm{O}_{2}$ sat using oximetry feedback. For adults with infrequent chest colds, rapid access to MIE during intercurrent infections may be all that is necessary.

\section{OUTCOMES}

An April 2010 consensus of clinicians from 22 centers reported $760 \mathrm{CNVS}$ dependent patients with NMD, none of whom could have survived more than minutes without ventilatory support $[55,56]$. Besides prolonging survival by CNVS rather than by CTMV, a controlled study also demonstrated 10 years longer survival by CNVS than by CTMV for DMD [57]. In another study all patients with a history of 1 month or more dependence on both CTMV and CNVS and with access to effective MIE preferred CNVS for safety, convenience, swallowing, speech, appearance, comfort, and overall [58]. Indeed, it would seem that no one with access to effective MIE and CNVS would ever want a tracheostomy tube nor would one be needed for survival other than for those with CNS or upper motor disorders.

Considering our patients with DMD, 101 eventually became CNVS-dependent for $7.4 \pm 6$.1 years to a mean $30.1 \pm 6.1$ years of age with 56 still alive. Twenty-six of the 101 became CNVS dependent without hospitalization or developing ARF. Eight CTMV users were decannulated to CNVS. Forty-five consecutive intubated DMD patients who could not pass spontaneous breathing trials before or after extubation were successfully extubated to CNVS and MIE. Seven have lived over age 40 including four who have required CNVS for 28, 19, 21, and 24 years to ages 41 , 44,48 , and 47 years. One is now 54 years old due to CNVS for over 30 years [59]. Eighteen other centers reported 284 CNVS dependent patients with DMD including many who were never hospitalized $[56,60,61]$. CNVS is also an alternative to tracheotomy in the perioperative management of children with flaccid neuromuscular scoliosis [62]. Since patients dependent on CTMV often die from complications related to the tracheostomy tubes, CTMV users with DMD are rarely reported to survive 40 years. I have seen only two.

Knowing that ALS patients who develop severe bulbarinnervated muscle impairment require tracheostomy tubes to survive, until 1995 I informed parents of children with SMA type 1 that unless they agreed to have their babies undergo tracheotomies that they die within one year. However, as can be seen from Fig. 3, I was wrong. The two boys whose parents I told this are now 24 and 21 years old and the latter has a 20 year old brother who has been CNVS dependent with no ventilator free breathing ability since 4 months of age. The two brothers have $0 \mathrm{~mL}$ of VC and no active muscle movement other than for extraoccular muscles. They became CNVS dependent without developing respiratory failure or requiring hospitalization. Each has been hospitalized for pneumonia once, at 8 and 12 years of age, respectively. They were intubated for 1 week, extubated back to CNVS, and returned directly home from the critical care unit. Thus, we learned that children with even the most severe SMAl can survive into adulthood using CNVS from infancy and even when ventilator unweanable can be extubated to CNVS and MIE without recourse to tracheotomy. We reported the successful extubation of 26 consecutive ventilator unweanable children with SMA1 in 2015 [46].

Considering patients with SMA1 who underwent tracheotomies before being referred to us, we reported 27 using CTMV for a mean of $6 \frac{1 / 2}{2}$ years and for up to 24 years from as early as 2 months of age. However, 25 of the 27 lost all ability to breathe immediately upon tracheotomy. None of the 21 who had not developed the ability to verbalize before undergoing tracheotomy did so after tracheotomy. By contrast, 72 SMAl patients using NVS are alive at a mean age of 86.1 months (range, 13-196 months); 13 out-of-state patients died at age 52.3 months (range, 13-111 months). Sixty-seven of the 75 could com- 
municate verbally. Eighteen SMA1 patients are now over 10 years old and seven over age 20 using CNVS without tracheostomy tubes and despite having $0 \mathrm{ml}$ of VC [63,64]. Others have also reported CNVS dependence for patients with SMA1 [65]. Five other centers reported 14 other CNVS dependent children with SMA1 [56]. Since even patients with the most severe SMA, type 1 that is, do not require tracheostomy tubes, those with milder SMAs and all other NMDs other than upper motor neuron disorders do not require them either.

Considering ALS we have now had 109 patients dependent on CNVS with 34 not having been hospitalized or developing respiratory failure despite becoming CNVS dependent. They used CNVS for about 10 months before developing upper airway spasticity that rendered MIE ineffective and necessitated tracheotomy for continued survival [10]. Ten other centers reported 226 CNVS dependent patients with ALS, also for a year or so with some patients relying on CNVS for up to 10 years before requiring tracheotomy [56]. Indeed, we have decannulated CTMV dependent non-bulbar ALS patients to CNVS, sparing them from invasive tubes for 6 months to up to 9 years by CNVS before they were to really require tracheostomy tubes for survival. Once upper motor neuron bulbar-innervated musculature became severely impaired and upper airway hypertonicity rendered MIE ineffective the ALS patients required tracheotomies for continued survival. However, several patients opted to die rather than undergo tracheotomy for a second time.

\section{DECANNULATION}

Since 1965 Dr. Augusta Alba, former medical director of GMH, included tracheostomy tube decannulation and transition from TMV to NVS as fundamental to the rehabilitation of ventilator users. In March of 1967 a 17-yearold boy fell off a gym horse at school to sustain C2 complete tetraplegia with immediate and permanent apnea and respirator dependence. Intubated then trached he remained in critical care for 10 months due to chronic lung infections. He was transferred to GMH for rehabilitation in February 1968 using CTMV with no ventilator free breathing ability. However, he developed severe trachiectasis with a cuff to trachea diameter ratio of 3:1. He continued to have severe airway mucus plugging that led to a respiratory arrest that resulted in cortical blind- ness and in two near arrests. He was motivated for tube removal, ostomy closure, and transition to CNVS which he prepared for by using mouthpiece NVS on pressure control $20 \mathrm{cmH}_{2} \mathrm{O}$ with the tracheostomy tube plugged in August 1968. He had begun iron lung use for sleep in July 1968. He learned and mastered GPB but could not use it effectively in the presence of the tracheostomy tube. After two more pneumonias his tracheostomy tube was removed and the ostomy closed in April 1969 and he was then able to effectively ventilate his lungs using GPB throughout daytime hours. His GPmaxSBC was $1,700 \mathrm{~mL}$ by comparison to a $\mathrm{VC}$ using accessory muscles in the sitting position of $420 \mathrm{~mL}$ [66]. Over the next 38 years he had only two episodes of pneumonia, neither of which necessitated intubation. Thus, he used mouthpiece NVS during the day and negative pressure ventilation (Pneumowrap; Lifecare Inc., Shelton, CT, USA) for sleep in a chronic care facility with no family contact until 2006 when depressed, blind, and with no functional limb movements, he requested and was withdrawn from ventilatory support.

Since this patient was initially placed in an iron lung in July 1969 and got used to it, he was never switched to lip cover or nasal NVS for sleep after I first described nasal NVS in 1987 [5]. Although the negative pressure ventilation caused obstructive apneas, he was not grossly symptomatic so he continued using it. In 1991 we reported having converted 37 negative pressure body ventilator users to sleep NVS and five of them came off of all antihypertensive medications. Their hypertension was cured by eliminating the obstructive apneas and repeated $\mathrm{O}_{2}$ desats caused by the negative pressure body ventilators [67].

Dr. Alba continued to decannulate TMV users, including patients with no ventilator free breathing ability, placing them on daytime mouthpiece NVS and lipseal NVS for sleep. We reported 257 up to CNVS mouthpiece NVS users in 1993 [28,39]. This included 25 high level SCI patients we decannulated to up to CNVS and reported in 1990 [28]. I was able to gather data on 20 mostly high level SCI patients decannulated to NVS by Dr. Viroslav and his group in Dallas, Texas [68]. He decannulated them to CNVS that included daytime mouthpiece NVS but had custom fabricated and fit thermoplastic oronasal interfaces constructed for sleep NVS rather than use simple lipseals [69]. It took Dr. Parker 10 hours to construct each one.

In 1996 we expanded decannulation from predomi- 
nantly SCI patients to decannulate 50 unweanable patients with NMD [70]. In preparation for decannulation we changed their tracheostomy tubes to cuffless fenestrated ones. First we accustomed the patients to speaking and maintain alveolar ventilation by using TMV with the cuffs deflated. We generally doubled the delivered volumes to typically $1,500 \mathrm{~mL}$ or more of air to compensate for air leakage up and around the tubes. This 'leaked air', about half of the delivered volume, permitted speech. The other half entered the lungs. No supplemental $\mathrm{O}_{2}$ was used during the preparation for decannulation. All $\mathrm{O}_{2}$ desats were reversed by using MIE via the tube, preferentially with the cuff inflated when one was present. Subsequently we discontinued TMV and capped the tubes so that the patients could practice mouthpiece NVS during the day then nasal NVS during sleep. Once mastering NVS-CNVS and MIE the tubes were changed to cuffless ones, capped permanently, and the patients used mouthpiece NVS during the day, sometimes intermittent abdominal pressure ventilators (IAPVs) [35], and lipseal or, after 1987 [5], nasal NVS for sleep. In the early years we removed the tubes in favor of tracheostomy buttons but since we never had to replace a tracheostomy after removing one we bypassed this step. The patients could practice 24 hour NVS using mouthpieces, IAPVs, and GPB during the day and lipseal, nasal, or oronasal interfaces for sleep as long as they liked. When confident of no longer needing the tracheostomy tubes we removed them. Airway suctioning was eliminated in favor of MIE via the invasive as well as the noninvasive interfaces including via simple mouthpieces.

Any patient, irrespective of extent of dependence on TMV, whose bulbar-innervated musculature is sufficient such that saliva aspiration does not cause a continuous decrease in baseline $\mathrm{O}_{2}$ sat below $95 \%$, and who has assisted CPF of at least $120 \mathrm{~L} / \mathrm{min}$ is a good candidate for decannulation to NVS [10]. In 2014 we reported the decannulation of 61 mainly unweanable CTMV users, 51 of whom in our outpatient clinic, after all had been comfortable using CNVS with the tubes capped for weeks to months [71]. As for the earlier studies, upon presentation the patients were placed on $\mathrm{FiO}_{2} 21 \%$ and $\mathrm{CO}_{2}$ was normalized by adjusting ventilator settings as needed. Then MIE was used via the tubes as much as needed, including up to every 30 minutes for periods, until ambient air $\mathrm{O}_{2}$ sat baseline remained greater than $94 \%$ and other decan- ulation criteria including having a normal white blood cell count, no signs of infection, discontinuance of any sedating medications, and full ability to cooperate were achieved. The patients were all decannulated to CNVS in ambient air as their care providers, who had been trained by our respiratory therapists, used MIE up to every 20 to 30 minutes to especially maintain $\mathrm{O}_{2}$ sat greater than $94 \%$ for the first 36 hours following decannulation.

Patients with tracheostomy tubes who had no CTMVfree breathing ability with VCs of $250 \mathrm{~mL}$ or greater invariably wean to less than continuous NVS after decannulation to it. Their VCs almost always increase and many wean to nocturnal-only NVS within 3 weeks of decannulation. The many reasons that TMV increases ventilator dependence have been summarized and include tube triggered airway secretions that block respiratory exchange membranes, and respiratory muscle deconditioning [52]. Tube removal also facilitates speech and swallowing. Thus, the lack of all ventilator free breathing ability does not mandate tracheostomy for any condition. Phrenic and diaphragm pacing should be limited only to high level SCI patients with no measurable VC and no ability to rotate the neck sufficiently to grab a mouthpiece with the lips and teeth for daytime CNVS. Even for such patients who can use pacing for daytime support, their tracheotomy tubes should be removed and NVS used for sleep to rest the electrically stimulated diaphragm and provide full ventilatory support while preventing the obstructive apneas that would occur with the use of pacing. Thus, only patients with severe upper motor neuron or CNS glottis dysfunction that results in $\mathrm{O}_{2}$ desat are poor candidates for decannulation $[10,11]$. As noted, all decannulated patients in one study preferred CNVS to CTMV for all issues surveyed [58].

\section{INTUBATION AND EXTUBATION OF VENTILATOR UNWEANABLE PATIENTS}

Conventional management with $\mathrm{O}_{2}$ and low span bilevel PAP instead of NVS and MIE must inevitably result in respiratory failure and intubation for patients with progressive muscle weakness. Once intubated for ARF, conventionally managed patients continue to receive supplemental $\mathrm{O}_{2}$ throughout the intubation period and during extubation attempts as well as following extubation even when their $\mathrm{O}_{2}$ sat levels would be absolutely 
normal without it. However, even an $\mathrm{FiO}_{2}$ of $25 \%$ can prevent airway secretion congestion or hypercapnia from hypoventilation from decreasing $\mathrm{O}_{2}$ sat below the normal baseline of $95 \%$ and so not alert the patient or clinician to mounting danger. If $\mathrm{CO}_{2}$ is not normal before extubation and ventilatory drive intact, if airway secretions are not effectively expulsed by using MIE via the translaryngeal tube, and if the lung pathology is not corrected prior to extubation as would be appreciated by a normalization of baseline $\mathrm{O}_{2}$ sat in $21 \% \mathrm{FiO}_{2}$, then the chances of extubation success are greatly diminished. It is for this reason that we rarely extubate anyone whose $\mathrm{O}_{2}$ sat in ambient air is less than $95 \%$. Likewise, while an infant can have a $\mathrm{PaCO}_{2}$ of $70 \mathrm{mmHg}$ and still have normal $\mathrm{O}_{2}$ sat, with age hypoventilation increasing $\mathrm{PaCO}_{2}$ to 47 to $55 \mathrm{mmHg}$ can result in $\mathrm{O}_{2}$ sat baseline decreasing below 95\%. Patients usually become symptomatic when hypercapnia is associated with decreases in daytime $\mathrm{O}_{2}$ sats below $95 \%$.

In 1978 at GMH I met 300 CNVS dependent patients. Many were CNVS dependent for over 20 years at that time and some are still alive and CNVS dependent today. I also witnessed numerous decannulations of CTMV dependent patients with little or no ventilator free breathing ability. In 1987 I reported the first patient to survive by using nasal CNVS despite having only $100 \mathrm{~mL}$ of VC [5]. I then recruited two CNVS dependent nocturnal lipseal NVS users with no measurable VC, one post-polio and one with high level SCI, to switch from lipseal to nasal NVS for one night. I observed them in their homes all night. Neither patient had any $\mathrm{O}_{2}$ desats nor any apparent arousals and both awoke refreshed in the morning. Thus, I realized then that nasal NVS could be used for full ventilatory support and that people with little or no respiratory muscle function did not need invasive airway tubes for it. Why, then, couldn't ventilator unweanable patients be safely extubated to CNVS?

With conventional critical care management, patients are ventilator weaned to be extubated. When intubated patients fail ventilator weaning parameters and spontaneous breathing trials or fail extubations to $\mathrm{O}_{2}$ and bi-level PAP they are told that tracheotomy is their only option for survival. However, ventilator weaning and passing spontaneous breathing trials are not prerequisites for successful extubation to CNVS and MIE. Patients can be extubated to 'wean' themselves rather than 'wean' to be extubated. Ideally, the respiratory muscle aids (NVS and
MIE) should have been introduced to patients and care providers in outpatient clinics and in the home before they required intubation so that they can be extubated to equipment at settings to which they are accustomed. Even when naïve of NVS and MIE, however, unweanable patients who meet criteria (Table 1) can be safely extubated to them without undergoing tracheotomy.

Conventional extubations are performed without supplemental $\mathrm{O}_{2}$ being turned off, hiding the fact that there might still be hypoventilation, airway mucus, and residual lung disease. Extubation to $\mathrm{O}_{2}$ therapy renders oximetry useless as a gauge of these three issues and the effectiveness of NVS and MIE to reverse them and it depresses ventilatory drive. Extubation to CPAP is useless and to low spans of bi-level PAP suboptimal for patients with respiratory muscle weakness. Bi-level PAP assists inspiratory efforts as a function of the absolute value of the drive pressure, that is, the IPAP EPAP difference. Patients who do not pass ventilator weaning or spontaneous breathing trials and ventilator weaning parameters or who fail extubation anyway need to be extubated to CNVS and MIE to succeed.

In 1988 we began to systematically extubate ventilator unweanable patients with ventilatory pump failure to

Table 1. Extubation criteria for ventilator unweanable patients

Fully alert and cooperative

Any sedative medications discontinued

Failure of respiratory function alone, other vital organs functional

Afebrile

Normal white blood cell count

Chest radiograph abnormalities cleared or clearing

Carbon dioxide tension $40 \mathrm{mmHg}$ or less at peak inspiratory pressures less than $35 \mathrm{cmH}_{2} \mathrm{O}$ on full ventilatory support settings with physiologic back-up rate as needed.

Oxyhemoglobin saturation $95 \%$ or higher for 12 hours or more in ambient air with all transient oxyhemoglobin desaturations below $95 \%$ reversed by mechanical insufflation-exsufflation via the translaryngeal tube

With translaryngeal tube cuff deflated, air leakage via upper airway sufficient for vocalization upon cuff deflation 
CNVS and MIE. Specific extubation criteria and a new extubation protocol were developed (Table 1). Once meeting the criteria, oro- or nasogastric tubes are removed to facilitate post-extubation nasal NVS. The patient is then extubated directly to CNVS on assist/control with volumes preset from $800-1,500 \mathrm{~mL}$ and rate, 10 to 14 per minute, and always in ambient air. The NVS is delivered via nasal, oronasal, and mouthpiece interfaces, usually nasal interfaces at first. Once successfully ventilated via nasal interface, they are taught mouthpiece NVS. This requires learning to use the soft palate to block air from leaking out of the nose. Finally, unless the patient had already been trained in LVR, they are taught to air stack via the mouthpiece or nasal interface.

Patients keep $15 \mathrm{~mm}$ angled mouthpieces accessible to their mouths (Fig. 1) and wean themselves, when possible, by taking fewer and fewer intermittent positive pressure ventilations, as tolerated. Diurnal nasal NVS is used for those who cannot grab and use a mouthpiece effectively. Generally, nasal or oronasal interfaces are used for nighttime NVS. With decreases in $\mathrm{O}_{2}$ sat below $95 \%$, ventilator positive inspiratory pressures, interface or tubing air leakage, $\mathrm{CO}_{2}$ retention, ventilator settings, and MIE use are possible etiologies to be corrected. Low ventilator positive inspiratory pressures indicate air leakage or inadequate settings. Most often, MIE is needed via oronasal interfaces at 50 to $60 \mathrm{cmH}_{2} \mathrm{O}$ pressures for decreases in $\mathrm{O}_{2}$ sat until the baseline returns to normal. Family members and other care providers remain with the patient in critical care for the first 36 hours post-extubation to apply the MIE and observe the oximetry for desats. Patients and care providers are also taught manually assisted coughing as well as MIE. Only the family and care providers will apply MIE up to every 20 to 30 minutes until the airway secretions diminish to pre-intubation levels and the $\mathrm{O}_{2}$ sat baseline remains normal. Care provider involvement can be critical both because the burden can be excessive for critical care staff and because the home care providers need to become proficient in MIE and NVS for the patient to go home safely and avoid future episodes of ARF.

When post-extubation oral intake is unsafe or inadequate and a gastrostomy tube is needed, this can be done without re-intubating the patient by interventional radiology [72], as an open gastrotomy by general surgery [73], or as a percutaneous endoscopic gastrostomy with the trochanter passed via an orifice in an oronasal interface that is also used to deliver NVS [71,74]. These approaches are summarized in my last book and in an updated version soon to be in press $[75,76]$.

In 2010 we reported extubating 157 consecutive 'unweanable' patients: 25 with SMA, 20 with DMD, 16 with ALS, 51 with other NMDs, 17 with spinal cord injury, and 11 PPSs. Eighty-three who refused tracheostomies were transferred from other hospitals. None could pass spontaneous breathing trials before or after extubation to CNVS and MIE. Extubation success was defined as not requiring reintubation during the hospitalization. Before hospitalization, 96 (61\%) patients had no experience with NVS, 41 (26\%) used it for less than CNVS, and 20 (13\%) were CNVS dependent. Our first-attempt protocol extubation success rate was $95 \%$ ( 149 patients). All 98 extubation attempts on patients with assisted CPF $160 \mathrm{~L} / \mathrm{min}$ or higher, as measured after extubation, were successful. Six of eight patients who failed an initial extubation attempt succeeded on subsequent attempts so only two bulbar ALS patients with no measurable assisted CPF underwent tracheotomy [45]. Since long-term chronic care facilities and nursing facility ventilator units lack expertise in noninvasive management we discharged these patients directly home even when no ventilator weaning was possible.

In 2015 we reported an additional 97 of 98 unweanable patients successfully extubated with 45 having been CNVS dependent for 4 months to 18 years before being intubated and the others being NVS naïve. Sixty-nine of the 98 were intubated for $24.9 \pm 22$ (range, 1-158) days and failed 0 to 6 (mean, 1.7) extubation attempts before being transferred and successfully extubated by us in $2.24 \pm 1.78$ (range, $<1-8$ ) days to CNVS. Two to 3 days was the usual time that it took for hourly MIE use via the translaryngeal tube to expulse airway secretions sufficiently for baseline $\mathrm{O}_{2}$ sat to normalize in ambient air. Normal baseline $\mathrm{O}_{2}$ sat is the primary criterion needed for extubation success. VC increased by $270 \%(\mathrm{p}<0.001)$ within 3 weeks following extubation. Weaning from CNVS to part-time NVS was achieved by all 52 subjects who had not been CNVS dependent before intubation. One subject underwent tracheotomy because of cardiovascular instability [46].

\section{CONCLUSION}

A simple evaluation designed to assess the respiratory 
muscle function of patients with ventilatory pump failure rather than a full battery of pulmonary function tests designed for obstructive/intrinsic lung disease, simple sleep $\mathrm{O}_{2}$ saturation and $\mathrm{CO}_{2}$ monitoring rather than polysomnography, and the application of pressures to the body and airways to support inspiratory and expiratory muscle function rather than supplemental oxygen therapy, CPAP, and bronchodilators can permit many progressively weakening and elderly patients to avoid ARF and tracheotomies. Those who do develop ARF, are intubated, and cannot pass spontaneous breathing trials can, nevertheless, be extubated, or even decannulated of tracheostomy tubes, to full-setting CNVS and MIE. Quality of life is optimized by noninvasive management and expense greatly reduced. Unfortunately, the conventional paradigm is that anyone who requires around-the-clock respiratory support needs a tracheostomy tube. Thus, a paradigm shift that is greatly disincentivized by the greater compensation for invasive procedures and management in general, is unlikely in the currently unsustainable American health care system. Noninvasive respiratory management is only desirable for patients and taxpayers, neither of whom have lobbyists for their interests.

\section{CONFLICT OF INTEREST}

No potential conflict of interest relevant to this article was reported.

\section{ACKNOWLEDGMENTS}

Patient consent was obtained for the use of the patient photos.

\section{REFERENCES}

1. Bach JR. Paradigm paralysis in the management of neuromuscular disease. J Med Pers 2013;11:24-9.

2. Bach JR. Amyotrophic lateral sclerosis. Communication status and survival with ventilatory support. Am J Phys Med Rehabil 1993;72:343-9.

3. Bach JR, Chiou M. Limitations of evidence-based medicine. Rev Port Pneumol 2016;22:4-5.

4. Lisboa C, Diaz O, Fadic R. Noninvasive mechanical ventilation in patients with neuromuscular diseases and in patients with chest restriction. Arch Bronco- neumol 2003;39:314-20.

5. Bach JR, Alba A, Mosher R, Delaubier A. Intermittent positive pressure ventilation via nasal access in the management of respiratory insufficiency. Chest 1987;92:168-70.

6. Mahajan KR, Bach JR, Saporito L, Perez N. Diaphragm pacing and noninvasive respiratory management of amyotrophic lateral sclerosis/motor neuron disease.

Muscle Nerve 2012;46:851-5.

7. Bach JR. Noninvasive respiratory management of high level spinal cord injury. J Spinal Cord Med 2012;35:7280.

8. Bolikal P, Bach JR, Goncalves M. Electrophrenic pacing and decannulation for high-level spinal cord injury: a case series. J Spinal Cord Med 2012;35:170-4.

9. Bach JR, Takyi SL. Physical medicine interventions to avoid acute respiratory failure and invasive airway tubes. PM R 2015;7:871-7.

10. Bach JR, Upadhyaya N. Association of need for tracheotomy with decreasing mechanical in-exsufflation flows in amyotrophic lateral sclerosis. Am J Phys Med Rehabil 2017 Apr 13 [Epub]. http://doi.org/10.1097/ PHM.0000000000000755.

11. Andersen T, Sandnes A, Brekka AK, Hilland M, Clemm $\mathrm{H}$, Fondenes $\mathrm{O}$, et al. Laryngeal response patterns influence the efficacy of mechanical assisted cough in amyotrophic lateral sclerosis. Thorax 2017;72:221-9.

12. Bach JR, Bianchi C, Aufiero E. Oximetry and indications for tracheotomy for amyotrophic lateral sclerosis. Chest 2004;126:1502-7.

13. Wade OL, Gilson JC. The effect of posture on diaphragmatic movement and vital capacity in normal subjects with a note on spirometry as an aid in determining radiological chest volumes. Thorax 1951;6:103-26.

14. Bach JR, Kang SW. Disorders of ventilation : weakness, stiffness, and mobilization. Chest 2000;117:3013.

15. Gay PC, Edmonds LC. Severe hypercapnia after lowflow oxygen therapy in patients with neuromuscular disease and diaphragmatic dysfunction. Mayo Clin Proc 1995;70:327-30.

16. Chiou M, Bach JR, Saporito LR, Albert O. Quantitation of oxygen-induced hypercapnia in respiratory pump failure. Rev Port Pneumol 2016;22:262-5.

17. Gomez-Merino E, Bach JR. Duchenne muscular dys- 
trophy: prolongation of life by noninvasive ventilation and mechanically assisted coughing. Am J Phys Med Rehabil 2002;81:411-5.

18. Bach JR, Alba AS. Management of chronic alveolar hypoventilation by nasal ventilation. Chest 1990;97:52-7.

19. Bach JR, Alba AS, Bodofsky E, Curran FJ, Schultheiss M. Glossopharyngeal breathing and noninvasive aids in the management of post-polio respiratory insufficiency. Birth Defects Orig Artic Ser 1987;23:99-113.

20. Won YH, Choi WA, Lee JW, Bach JR, Park J, Kang SW. Sleep transcutaneous vs. end-tidal $\mathrm{CO}_{2}$ monitoring for patients with neuromuscular disease. Am J Phys Med Rehabil 2016;95:91-5.

21. Cinel D, Markwell K, Lee R, Szidon P. Variability of the respiratory gas exchange ratio during arterial puncture. Am Rev Respir Dis 1991;143:217-8.

22. Bach JR, Bianchi C, Vidigal-Lopes M, Turi S, Felisari G. Lung inflation by glossopharyngeal breathing and "air stacking" in Duchenne muscular dystrophy. Am J Phys Med Rehabil 2007;86:295-300.

23. Dail C, Rodgers M, Guess V, Adkins HV. Glossopharyngeal breathing. Downey: Rancho Los Amigos Hospital, Department of Physical Therapy; 1979.

24. Dail CW, Affeldt JE. Glossopharyngeal breathing [Video]. Los Angeles: College of Medical Evangelists, Department of Visual Education; 1954.

25. Webber B, Higgens J. Glossopharyngeal breathing what, when and how? [Video]. West Sussex: Aslan Studios Ltd.; 1999.

26. Chiou M, Bach JR, Jethani L, Gallagher MF. Active lung volume recruitment to preserve vital capacity in Duchenne muscular dystrophy. J Rehabil Med 2017;49:49-53.

27. Kang SW, Bach JR. Maximum insufflation capacity. Chest 2000;118:61-5.

28. Bach JR, Alba AS. Noninvasive options for ventilatory support of the traumatic high level quadriplegic patient. Chest 1990;98:613-9.

29. Bach JR, Bianchi C. Prevention of pectus excavatum for children with spinal muscular atrophy type 1 . Am J Phys Med Rehabil 2003;82:815-9.

30. McKim DA, Griller N, LeBlanc C, Woolnough A, King J. Twenty-four hour noninvasive ventilation in Duchenne muscular dystrophy: a safe alternative to tracheostomy. Can Respir J 2013;20:e5-9.

31. Suri P, Burns SP, Bach JR. Pneumothorax associated with mechanical insufflation-exsufflation and related factors. Am J Phys Med Rehabil 2008;87:951-5.

32. Bach JR, Mahajan K, Lipa B, Saporito L, Goncalves M, Komaroff E. Lung insufflation capacity in neuromuscular disease. Am J Phys Med Rehabil 2008;87:720-5.

33. Ishikawa Y. Manual for the care of patients using noninvasive ventilation. Matsudo: Japan Planning Center; 2005.

34. Bach JR, Alba AS, Shin D. Management alternatives for post-polio respiratory insufficiency. Assisted ventilation by nasal or oral-nasal interface. Am J Phys Med Rehabil 1989;68:264-71.

35. Bach JR, Alba AS. Intermittent abdominal pressure ventilator in a regimen of noninvasive ventilatory support. Chest 1991;99:630-6.

36. Bach JR, Radbourne M. A mechanical intermittent abdominal pressure ventilator. Am J Phys Med Rehabil. Forthcoming 2017.

37. Allen J. Pulmonary complications of neuromuscular disease: a respiratory mechanics perspective. Paediatr Respir Rev 2010;11:18-23.

38. Bach JR, Robert D, Leger P, Langevin B. Sleep fragmentation in kyphoscoliotic individuals with alveolar hypoventilation treated by NIPPV. Chest 1995;107:1552-8.

39. Bach JR, Alba AS, Saporito LR. Intermittent positive pressure ventilation via the mouth as an alternative to tracheostomy for 257 ventilator users. Chest 1993;103:174-82.

40. Richards GN, Cistulli PA, Ungar RG, Berthon-Jones M, Sullivan CE. Mouth leak with nasal continuous positive airway pressure increases nasal airway resistance. Am J Respir Crit Care Med 1996;154:182-6.

41. Morelot-Panzini C, Le Pimpec-Barthes F, Menegaux F, Gonzalez-Bermejo J, Similowski T. Referred shoulder pain (C4 dermatome) can adversely impact diaphragm pacing with intramuscular electrodes. Eur Respir J 2015;45:1751-4.

42. DiPALS Writing Committee. Safety and efficacy of diaphragm pacing in patients with respiratory insufficiency due to amyotrophic lateral sclerosis (DiPALS): a multicentre, open-label, randomised controlled trial. Lancet Neurol 2015;14:883-92.

43. DeLisa JA, Bach JR. Overview of rehabilitation, general evaluation principles and the rehabilitation team. In: Bach JR, editor. Pulmonary rehabilitation: the ob- 
structive and paralytic conditions. Philadelphia: Hanley \& Belfus; 1996. p. 13-25.

44. Guerin C, Bourdin G, Leray V, Delannoy B, Bayle F, Germain M, et al. Performance of the coughassist insufflation-exsufflation device in the presence of an endotracheal tube or tracheostomy tube: a bench study. Respir Care 2011;56:1108-14.

45. Bach JR, Goncalves MR, Hamdani I, Winck JC. Extubation of patients with neuromuscular weakness: a new management paradigm. Chest 2010;137:1033-9.

46. Bach JR, Sinquee DM, Saporito LR, Botticello AL. Efficacy of mechanical insufflation-exsufflation in extubating unweanable subjects with restrictive pulmonary disorders. Respir Care 2015;60:477-83.

47. Fishburn MJ, Marino RJ, Ditunno JF Jr. Atelectasis and pneumonia in acute spinal cord injury. Arch Phys Med Rehabil 1990;71:197-200.

48. Garstang SV, Kirshblum SC, Wood KE. Patient preference for in-exsufflation for secretion management with spinal cord injury. J Spinal Cord Med 2000;23:805.

49. Bach JR, Smith WH, Michaels J, Saporito L, Alba AS, Dayal R, et al. Airway secretion clearance by mechanical exsufflation for post-poliomyelitis ventilator-assisted individuals. Arch Phys Med Rehabil 1993;74:170-7.

50. Barach AL, Beck GJ. Exsufflation with negative pressure; physiologic and clinical studies in poliomyelitis, bronchial asthma, pulmonary emphysema, and bronchiectasis. AMA Arch Intern Med 1954;93:825-41.

51. Bach JR. Mechanical insufflation-exsufflation: comparison of peak expiratory flows with manually assisted and unassisted coughing techniques. Chest 1993;104:1553-62.

52. Bach JR. Conventional approaches to managing neuromuscular ventilation failure. In: Bach JR, editor. Pulmonary rehabilitation: the obstructive and paralytic conditions. Philadelphia: Hanley \& Belfus; 1996. p. 285-301.

53. Bach JR, Rajaraman R, Ballanger F, Tzeng AC, Ishikawa $Y$, Kulessa R, et al. Neuromuscular ventilatory insufficiency: effect of home mechanical ventilator use v oxygen therapy on pneumonia and hospitalization rates. Am J Phys Med Rehabil 1998;77:8-19.

54. Toussaint M, Steens M, Wasteels G, Soudon P. Diurnal ventilation via mouthpiece: survival in end-stage
Duchenne patients. Eur Respir J 2006;28:549-55.

55. Bach JR, Goncalves MR, Hon A, Ishikawa Y, De Vito EL, Prado F, et al. Changing trends in the management of end-stage neuromuscular respiratory muscle failure: recommendations of an international consensus. Am J Phys Med Rehabil 2013;92:267-77.

56. Goncalves MR, Bach JR, Ishikawa Y, Saporito, Winck JC. Continuous noninvasive ventilatory support outcomes for neuromuscular disease: a multicenter collaboration and literature review. Rev Port Pneumol. Forthcoming 2017.

57. Ishikawa $\mathrm{Y}$, Miura T, Ishikawa $\mathrm{Y}$, Aoyagi T, Ogata $\mathrm{H}$, Hamada S, et al. Duchenne muscular dystrophy: survival by cardio-respiratory interventions. Neuromuscul Disord 2011;21:47-51.

58. Bach JR. A comparison of long-term ventilatory support alternatives from the perspective of the patient and care giver. Chest 1993;104:1702-6.

59. Villanova M, Kazibwe S. New survival target for Duchenne muscular dystrophy. Am J Phys Med Rehabil 2017;96:e28-e30.

60. Kohler M, Clarenbach CF, Boni L, Brack T, Russi EW, Bloch KE. Quality of life, physical disability, and respiratory impairment in Duchenne muscular dystrophy. Am J Respir Crit Care Med 2005;172:1032-6.

61. Villanova M, Brancalion B, Mehta AD. Duchenne muscular dystrophy: life prolongation by noninvasive ventilatory support. Am J Phys Med Rehabil 2014;93:595-9.

62. Mills B, Bach JR, Zhao C, Saporito L, Sabharwal S. Posterior spinal fusion in children with flaccid neuromuscular scoliosis: the role of noninvasive positive pressure ventilatory support. J Pediatr Orthop 2013;33:488-93.

63. Bach JR, Tuccio MC, Khan U, Saporito LR. Vital capacity in spinal muscular atrophy. Am J Phys Med Rehabil 2012;91:487-93.

64. Bach JR, Gupta K, Reyna M, Hon A. Spinal muscular atrophy type 1: prolongation of survival by noninvasive respiratory aids. Pediatr Asthma Allergy Immunol 2009;22:151-62.

65. Schroth MK. Special considerations in the respiratory management of spinal muscular atrophy. Pediatrics 2009;123 Suppl 4:S245-9.

66. Bach JR. Case studies of respiratory management. In: Bach JR, editor. Pulmonary rehabilitation: the ob- 
structive and paralytic conditions. Philadelphia: Hanley \& Belfus; 1996. p. 331-46.

67. Bach JR, Penek J. Obstructive sleep apnea complicating negative-pressure ventilatory support in patients with chronic paralytic/restrictive ventilatory dysfunction. Chest 1991;99:1386-93.

68. Bach JR. New approaches in the rehabilitation of the traumatic high level quadriplegic. Am J Phys Med Rehabil 1991;70:13-9.

69. McDermott I, Bach JR, Parker C, Sortor S. Customfabricated interfaces for intermittent positive pressure ventilation. Int J Prosthodont 1989;2:224-33.

70. Bach JR, Saporito LR. Criteria for extubation and tracheostomy tube removal for patients with ventilatory failure: a different approach to weaning. Chest 1996;110:1566-71.

71. Bach JR, Saporito LR, Shah HR, Sinquee D. Decanulation of patients with severe respiratory muscle insufficiency: efficacy of mechanical insufflation-exsufflation. J Rehabil Med 2014;46:1037-41.

72. Chesoni SA, Bach JR, Okamura EM. Massive reflux and aspiration after radiographically inserted gastrostomy tube placement. Am J Phys Med Rehabil 2015;94:e6-9.

73. Bach JR, Gonzalez M, Sharma A, Swan K, Patel A. Open gastrostomy for noninvasive ventilation users with neuromuscular disease. Am J Phys Med Rehabil 2010;89:1-6.

74. Banfi P, Volpato E, Valota C, D'Ascenzo S, Bani Alunno C. Noninvasive ventilation during feeding tube placement. Respir Care. Forthcoming 2017.

75. Ramalho RM, Coelho R, Bach JR. Gastrointestinal complications and enteral nutrition. In: Bach JR, editor. Physical medicine and rehabilitation interventions for skeletal and respiratory muscle dysfunction. [place unknown]: VentilaMed BreatheNVS. Forthcoming 2017.

76. Swan K, Kazanjian MS, Gulyas A. Swallowing and gastrointestinal concerns. In: Bach JR, editor. Management of patients with neuromuscular disease. Philadelphia: Hanley \& Belfus; 2004. p. 51-74. 